\title{
The mechanisms of muscle wasting in COPD and heart failure
}

\section{Meccanismi della miopatia scheletrica nella broncopneumopatia cronica ostruttiva e nello scompenso cardiaco}

\section{Giorgio Vescovo*}

\author{
Department of Internal Medicine, San Bortolo Hospital, Vicenza, Italy
}

Received 19 May 2011; accepted 18 July 2011

available online 7 September 2011

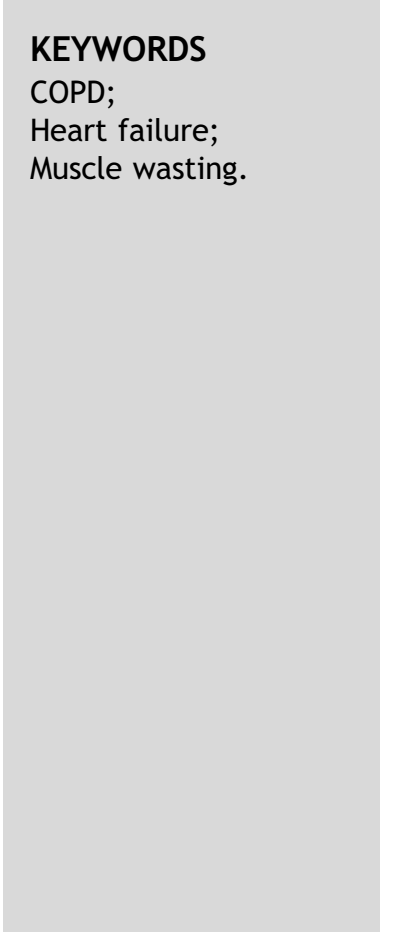

Summary Many of the mechanisms leading to skeletal muscle wasting in COPD and heart failure are common to both conditions. These encompass neurohormonal activation and systemic inflammation.

The mechanisms leading to muscle dysfunction are both qualitative and quantitative. Qualitative changes comprise the transition from aerobic metabolism and prevalent slow fibers composition toward anaerobic metabolism and fast fibers synthesis. Quantitative changes are mainly linked to muscle loss. These changes occur not only in the major muscles bulks of the body but also in respiratory muscles.

The mechanisms leading to muscle wastage include cytokine-triggered skeletal muscle apoptosis and ubiquitin-proteasomeand non-ubiquitin-dependent pathways.

The regulation of fiber type involves the growth hormone/insulin-like growth factor $1 /$ calcineurin/transcriptional coactivator PGC1 cascade. The imbalance between protein synthesis and degradation plays an important role. Protein degradation can occur through ubiquitindependent and non-ubiquitin-dependent pathways. Very recently, two systems controlling ubiquitin-proteasome activation have been described: FOXO-ubiquitin ligase and NFkB ubiquitin ligase. These are triggered by TNF $\alpha$ and growth hormone/insulin-like growth factor 1 .

Moreover, apoptosis, which is triggered by tumor necrosis factor $\alpha$, plays an important role. Another mechanism acting on muscle wastage is malnutrition, with an imbalance between catabolic and anabolic factors toward the catabolic component. Catabolism is also worsened by the activation of the adrenergic system and alteration of the cortisol/DEHA ratio toward cortisol production. Sarcomeric protein oxidation and its consequent contractile impairment can be another cause of skeletal muscle dysfunction in CHF.

(c) 2011 Elsevier Srl. All rights reserved.

\footnotetext{
* Corresponding author. San Bortolo Hospital, viale Rodolfi 37 - 36100 Vicenza, Italy.

E-mail: gvescovo@yahoo.it (G. Vescovo).
} 


\section{Introduction}

The purpose of this review is to elucidate the mechanisms of skeletal muscle dysfunction and wasting in chronic diseases characterized by neurohormonal activation and systemic inflammation, such as COPD and heart failure (Fig. 1). COPD is a major cause of death and disability worldwide. Treatment of COPD improves lung function but is unlikely to slow the steady downhill course of the disease or reduce mortality. In COPD, numerous abnormalities can be found outside the lung. These include systemic inflammation, cachexia, and skeletal muscle dysfunction. Thus, COPD has been called a systemic disease. Convincing data have demonstrated that COPD causes neurohumoral activation. Based on experience with chronic heart failure and other diseases characterized by neurohumoral activation, we hypothesize that the negative consequences of neurohumoral activation, namely inflammation, cachexia, effects on ventilation, and skeletal muscle dysfunction, give rise to a self-perpetuating cycle that contributes to the pathogenesis of COPD and may involve respiratory muscle dysfunction and systemic inflammation. This concept may help to further explain the increased cardiovascular morbidity and mortality in COPD patients [1-9]. CHF shares many pathophysiological aspects with COPD, from systemic inflammation to hormonal disorders and muscle dysfunction and wastage.
In these conditions, qualitative and quantitative mechanisms lead to muscle dysfunction. Qualitative changes encompass the transition from aerobic metabolism and prevalent slow fibers composition toward anaerobic metabolism and fast fibers synthesis, whereas quantitative changes are mainly linked to muscle loss [10-13]. These changes occur not only in the major muscles bulks of the body but also in respiratory muscles [14]. The muscle hypothesis of heart failure also suggests that symptoms such as dyspnea and fatigue are partially due to skeletal muscle alterations. Hyperventilation due to an altered ergoreflex seems to contribute to shortness of breath [15]. Mechanisms leading to muscle wastage include cytokine-triggered skeletal muscle apoptosis and ubiquitin-proteasome and non-ubiquitin-dependent pathways. The regulation of fiber type involves the growth hormone/insulin-like growth factor $1 /$ calcineurin/transcriptional coactivator PGC1 cascade [16]. The imbalance between protein synthesis and degradation plays an important role. Protein degradation can occur through ubiquitin-dependent and non-ubiquitin-dependent pathways. Very recently, two systems controlling ubiquitinproteasome activation have been described: FOXO-ubiquitin ligase and NFkB ubiquitin ligase [13]. These are triggered by $\mathrm{TNF} \alpha$ and growth hormone/ insulin-like growth factor 1 $[17,18]$. However, apoptosis also plays an important role. In humans and experimental models of heart failure,
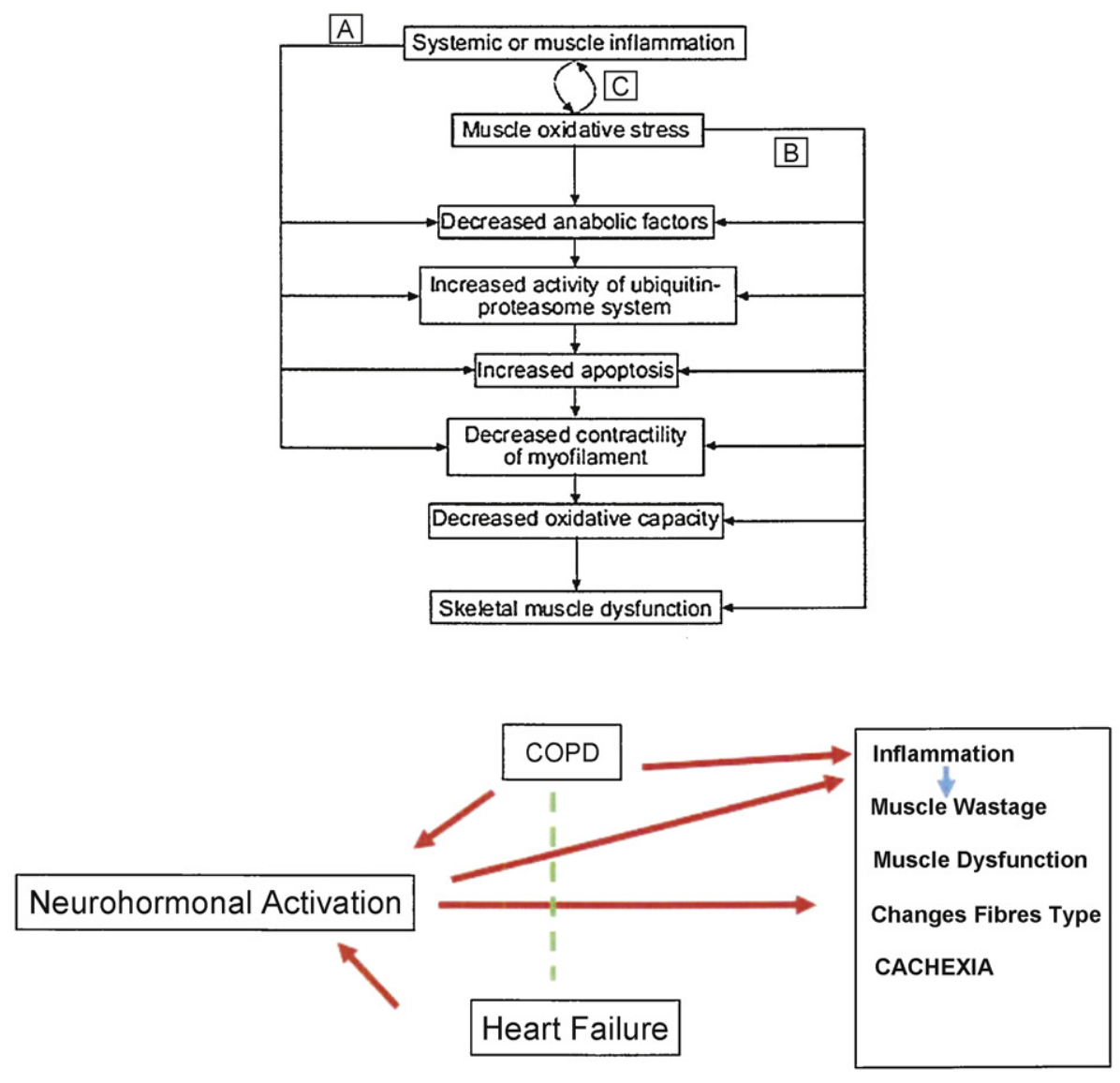

Figure 1 Mechanisms of muscle wastage in COPD and Heart Failure. 
programmed cell death has been found in skeletal muscle and interstitial cells. Apoptosis is triggered by tumor necrosis factor $\alpha$, and in-vitro experiments have shown that it can be induced by its second messenger, sphingosine (SPH). Apoptosis correlates with the severity of the heart failure syndrome. It involves activation of caspases 3 and 9 and mitochondrial cytochrome c release [10,12]. Another mechanism acting on muscle wastage is malnutrition, with an imbalance between catabolic and anabolic factors toward the catabolic component. Catabolism is also worsened by the activation of the adrenergic system and by the alteration of the cortisol/DEHA ratio toward cortisol production. Sarcomeric protein oxidation and its consequent contractile impairment can be another cause of skeletal muscle dysfunction in CHF [19].

\section{Qualitative changes in skeletal muscle}

(Fig. 2 A-B)

The major changes occurring in the skeletal muscles of patients and animals with COPD and CHF are consistent with a shift from slow aerobic fibers to fast fibers. The metabolic features of slow fibers are as follows: abundance of mitochondria and prevalence of slow myosin heavy chains (MHCs), characterizing anaerobic metabolism.

\section{Myosin heavy chain composition and fiber type} (Fig. 2C)

Skeletal muscle fiber type is determined by the myosin heavy chain pattern. There are three major myosin heavy chains (MHCs) that can be separated electrophoretically on the basis of their relative mobility. MHC1 is the slow anaerobic isoform, characterized by low ATP consumption and low shortening speed and defined as fatigue resistant. Type I fibers are mainly composed of MHC1. MHC2a is the fast oxidative component and $\mathrm{MHC} 2 \mathrm{~b}$ is the fast glycolytic. They possess higher ATP consumption and higher shortening speeds, and they both are more fatigable because they reach the anaerobic threshold earlier. Types $\mathrm{Ila}$ and IIb are mainly composed of $\mathrm{MHC} 2 \mathrm{a}$ and $\mathrm{MHC} 2 \mathrm{~b}$, respectively. In the gastrocnemius of patients with $\mathrm{CHF}$, there is a shift from slow to fast isoforms [11]. The magnitude of this shift correlates with the indices of CHF syndrome severity, such as the NYHA class, exercise test tolerance measured in minutes, and diuretic consumption. This myopathy is generalized and involves the diaphragmatic muscle [14]. Skeletal muscle composition is in some way responsible for the reduced exercise capacity in patients with COPD and CHF. MHC composition correlates with peak $\mathrm{VO}_{2}$, VT and $\mathrm{O}_{2}$ pulse, with a strong positive correlation between $\mathrm{MHC} 1$ and peak $\mathrm{VO}_{2}$, VT and $\mathrm{O}_{2}$ pulse, suggesting that a high percentage of glycolytic fibers reduces exercise capacity because of the early appearance of anaerobic metabolism.

\section{Muscle atrophy and changes in fiber type and MHC composition}

The origin of this myopathy has been long debated. Atrophy due to deconditioning has been thought to play a role in the genesis of this myopathy, but muscle bulk loss due to extreme inactivity is accompanied by increased expression of MHC1 [13]. The debate on the possible causes of this myopathy is still open. It has been hypothesized that cytokine activation and loss of anabolic function, ergometaboloreceptor dysfunction or changes in blood flow may be of importance [15].

The shift toward fast isomyosins occurs both in fast and slow muscles (soleus and EDL) and is due to specific increased activity of mRNA encoding MHC2b rather than specific atrophy of type I fibers. Pro-apoptotic caspase-3 significantly increases, while $\mathrm{Bcl}-2$, which is protective against apoptosis, drops significantly [21]. Apoptosis (Fig. 3 A-B) is also responsible for the appearance of muscle atrophy in fast muscles, as demonstrated by the reduced muscle weight/body weight ratio and fiber cross-sectional area. We also showed [21] that atrophy is preceded by the shift toward fast isoforms, again confirming that biochemical changes are independent of muscle wasting. Apoptosis in the skeletal muscle appears simultaneously with worsening CHF and occurs parallel with increased levels of circulating TNF. This cytokine is known to produce muscle wasting by increasing apoptosis [12] and activating ubiquitin, thereby triggering the protein-waste pathway [22]. In CHF, endothelial cells show [20] a very high degree of apoptosis, which, even in the absence of changes in skeletal muscle blood flow, could alter myofiber nutrition. Decreased oxygen delivery through a damaged or even decreased capillary network can cause relative ischemia, which muscle could adapt to by increasing the synthesis of $2 \mathrm{~b}$ anaerobic fibers. When ischemia is more pronounced, fibers can be irreversibly damaged and undergo apoptosis, which ultimately leads to muscle loss and atrophy.

\section{Determination of fiber type (Fig. 4)}

The synthesis of slow fibers is regulated by calcineurin and mitogen-activated protein kinase as well as TNFalpha-activated nuclear factor kappaB [23]. In CHF myopathy, a shift from slow-oxidative to fast-glycolytic fibers has been described by several authors and is a cause of limited exercise capacity. The transition from slow to fast fibers is controlled by the FK506-FK506-binding protein complex (cyclosporine receptor) and calcineurin [24,25]. It is possible that IGF1 overexpression due to growth hormone treatment may produce a re-shift from fast to slow fibers in heart failure. This has been demonstrated by Dalla Libera et al. [18] in an animal model of CHF, and in the future, it may be a therapeutic target for improving muscle mass and function.

\section{Quantitative muscle changes and mechanisms controlling fiber growth and size (Fig. 4)}

\section{Growth hormone/insulin-like growth factor 1 resistance}

Growth hormone/insulin-like growth factor 1 (IGF1) resistance has been considered to be one of the most important mechanisms leading to muscle atrophy [26]. IGF1 can stimulate skeletal muscle growth by activating phosphoinositide 3-kinase, which in turn phosphorylates serine/threonine 

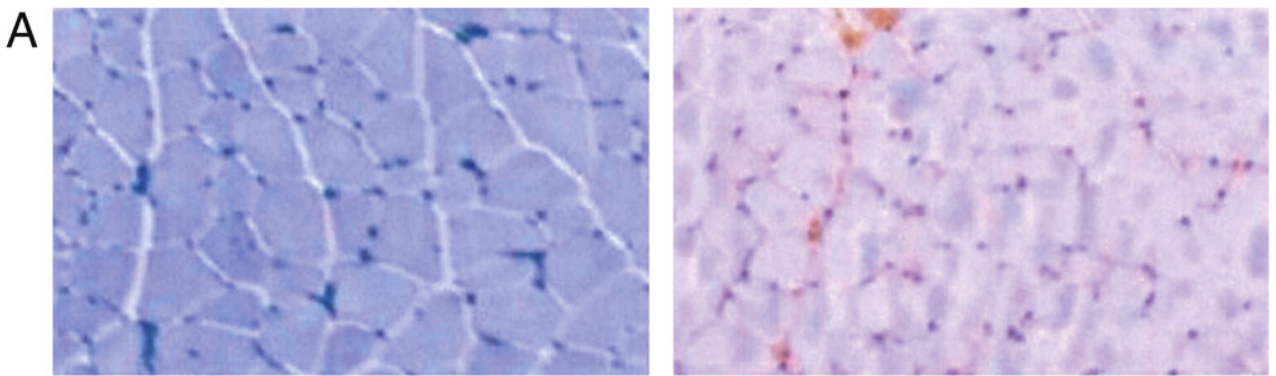

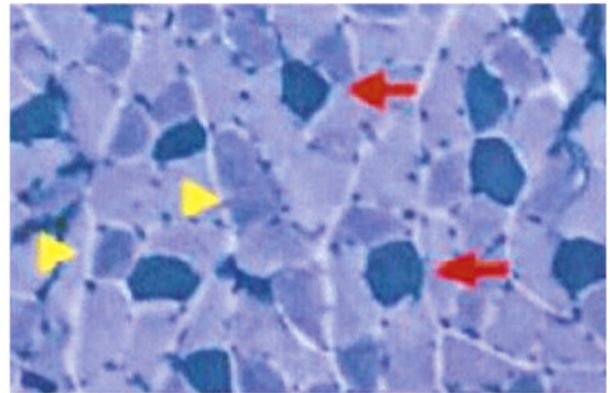

Metachromatic stain

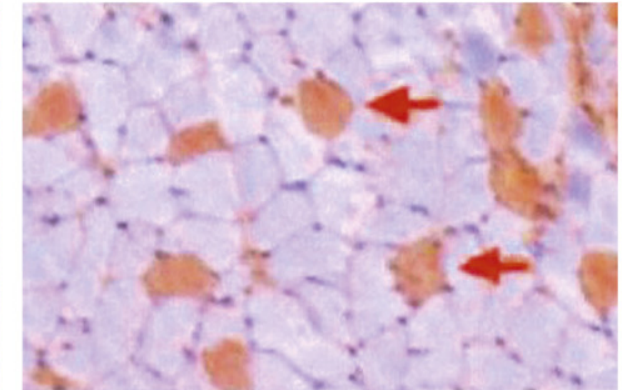

Anti-myosin (slow)

b
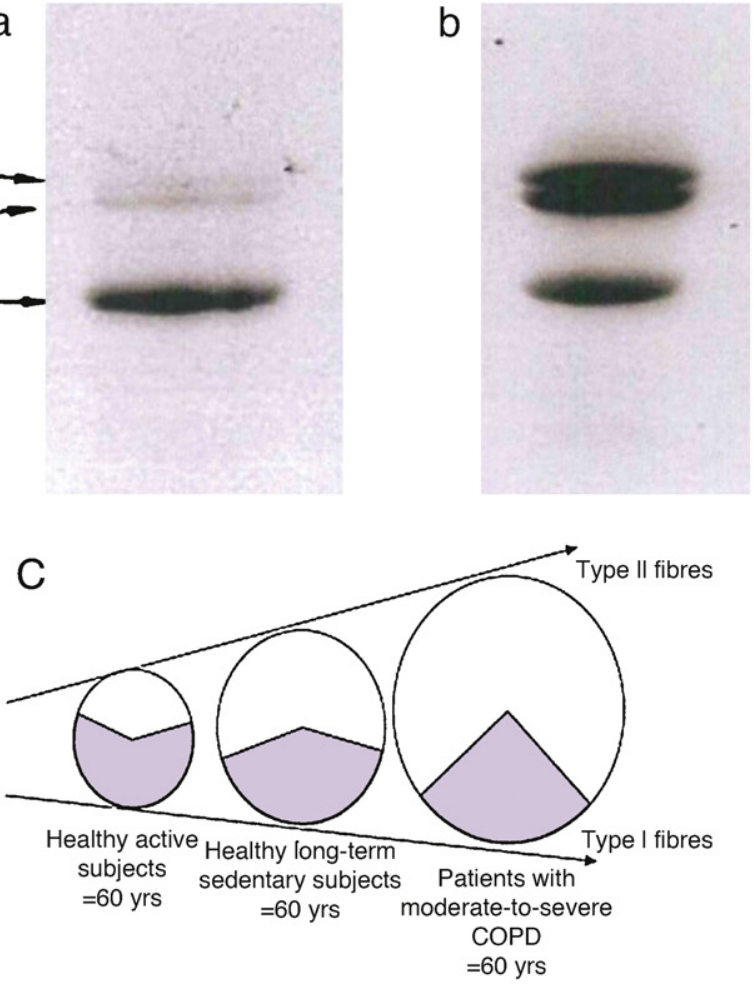

Figure 2 A-B Differences between Fast and Slow fibers detected with metachromatic stain and anti-myosin antibodies. Bottom panel shows the electrophoretical separation of fast (MHC2a and MHC2b) and slow isoforms (MHC1). A control subject, B patient with shift toward slow myosins. 2C Distribution of muscle fibres type in COPD patients according to disease severity.

kinase AKT, a powerful activator of the mammalian target of rapamycin (mTOR) that stimulates protein synthesis. Phosphorylated AKT inhibits the activity of FOXO, a member of the forkhead receptor superfamily and a transcription factor that activates the expression of atrogin-1 (ubiquitin ligase) and atrogenes and leads to protein degradation. When cachexia develops, FOXO is activated with atrogin-1 and atrogenes, and protein degradation occurs [24,27,28,30]. The overexpression of IGF1 and the administration of growth hormone can prevent muscle atrophy, which according to 

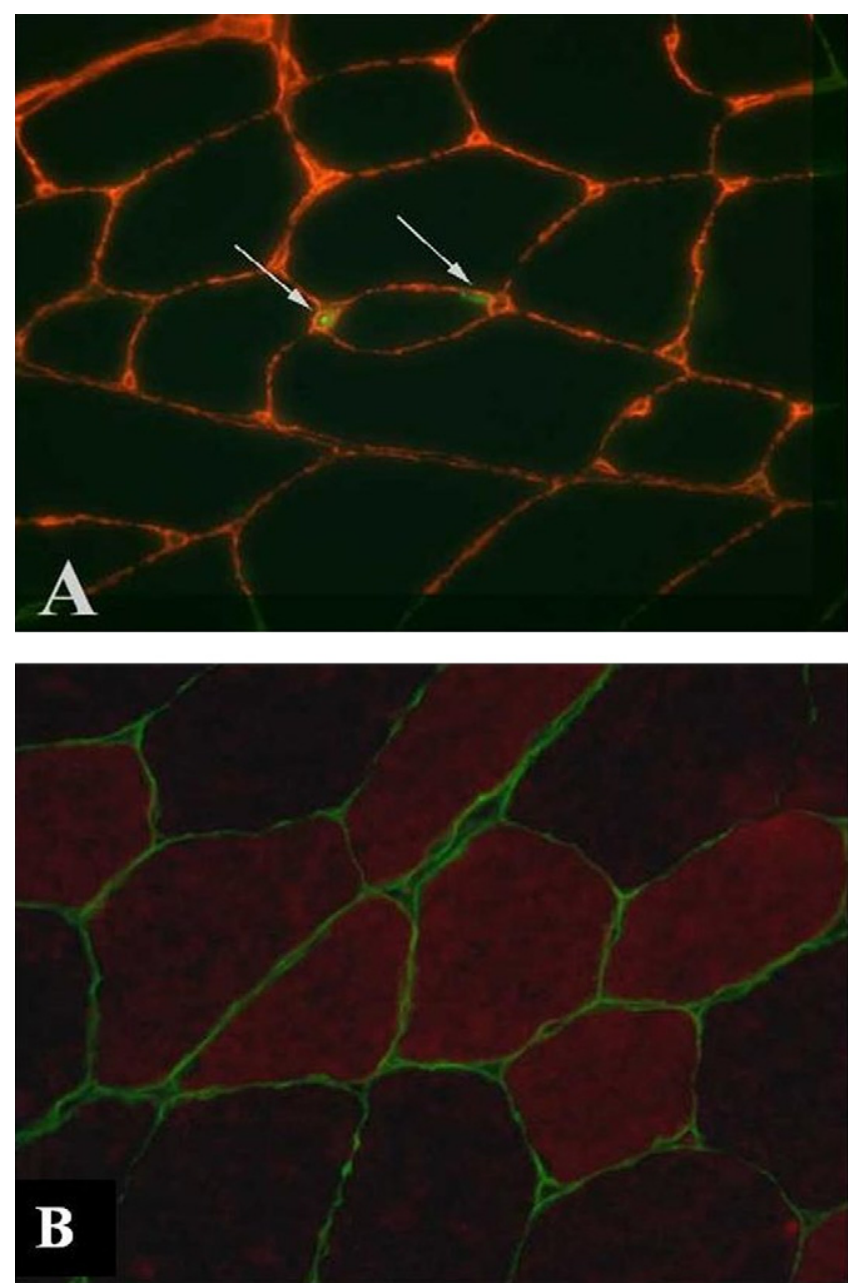

Figure 3 A-B Skeletal muscle fibers undergoing apoptosis. TUNEL positivity is seen in panel A. Panel B shows presence of apoptosis both in "fast" and "slow" fibers (double staining for laminin and antibodies against MHC1).

the present observations can be ascribed to the inhibition of FOXO and activation of mTOR $[29,30]$. Growth hormone administration can prevent muscle atrophy by blocking the activated caspase system and consequent apoptotic nuclear death [18]. This confirms the broad overlap existing between pathways such as apoptosis and protein wastage. Growth hormone/IGF1 axis stimulation also leads to the activation of transcriptional mechanisms that can induce the qualitative changes in skeletal muscle fiber composition described above. IGF1 leads to calcineurin expression, which in turn regulates the intracellular expression of peroxisome proliferator-activated receptor-coactivator PGC1 $\alpha$ [31]. This can activate mitochondrial biogenesis and oxidative metabolism and is the principal factor regulating muscle fiber type [24,25]. Calcineurin in skeletal muscle co-regulates the contractile and metabolic components of the slow-muscle phenotype and promotes transcription and muscle remodeling. This is achieved by the activation of $\mathrm{PGC} 1 \alpha$, a nuclear factor of activated T cells and nuclear respiratory factors 1 and 2 . Cyclosporin A and FK506 can block this pathway. PGC1a provides a plausible molecular basis for the connection between environmental-hormonal stimulation (catecholamines and cytokines) and mitochondrial biogenesis. The FOXO system is controlled by extracellular stimuli, such as contraction, insulin, angiotensin II, pressure and volume overload.

\section{Muscle Wasting}

The mechanisms leading to muscle wasting are complex and not univocal. They comprise apoptosis, an imbalance between protein synthesis and degradation, exaggerated protein degradation, and catabolic-anabolic imbalance.

\section{Apoptosis (Fig. 3 A-B)}

It is clear that body weight loss is mainly due to loss in lean mass. However, how this outcome is reached is complicated and unclear. Moreover, there are several mechanisms involved in muscle wasting.

The role played by hormones and the catabolic-anabolic imbalance has been described. There are many other factors that can cause muscle wasting, such as decreased caloric intake, altered gut absorption, and reduced peripheral flow, but pro-inflammatory cytokines play a key role in muscle atrophy development. Muscle atrophy can be detected by a computerized tomography scan, dual energy X-ray absorptiometry, and measurements of fiber cross-sectional areas on skeletal muscle biopsies. The severity of $\mathrm{CHF}$ and exercise capacity is measured as the maximal $\mathrm{O}_{2}$ consumption, which correlates with the degree of muscle atrophy [32]. The number of apoptotic nuclei correlates with the degree of muscle atrophy and the circulating levels of TNF $\alpha$ and its second messenger, SHP $[10,12,21]$. In vitro experiments have demonstrated that SPH can induce apoptosis in a dose-dependent fashion in cultured myotubes [10]. We have therefore postulated that apoptosis can play a role in determining muscle atrophy. When apoptosis occurs in multinucleated skeletal muscle fibers with compartmentalized metabolism they develop atrophy rather than cell death because they lose only a part of themselves Ubiquitin levels are often increased in cachexia (Fig. 5), also suggesting that TNF $\alpha$ can also determine atrophy via TNF $\alpha$-ubiquitin dependent protein wastage $[22,34,35]$. We do not know whether TNF $\alpha$ can induce apoptosis directly on skeletal muscle. We tend to hypothesize that TNF $\alpha$ acts through its second messenger, SPH. TNF $\alpha$, via the TNFR1 receptor located on the heart myocytes, activates FAN (factor activating neutral sphingomyelinase) and subsequently sphingomyelinase, which mediates the production of ceramide and SPH $[33,34]$. These sphingolipids are released into the blood stream and can induce skeletal muscle apoptosis.

At the muscle level, TUNEL positivity, which is a feature of nuclear strand breaks, is accompanied by caspase 3 and 9 activation, decreased levels of Bcl2 and increased levels of Bax [13]. Cytochrome C is increased in the cytosol of apoptotic cells, confirming that the mitochondrial pathway of apoptosis is activated.

Another mechanism that can lead to muscle apoptosis and muscle wasting is the activation of oxidative stress with production of iNOS [36]. There are also recent observations that nitric oxide can be generated by the interaction of sphingolipids at a transcriptional level with the involvement of NFKB [37]. 


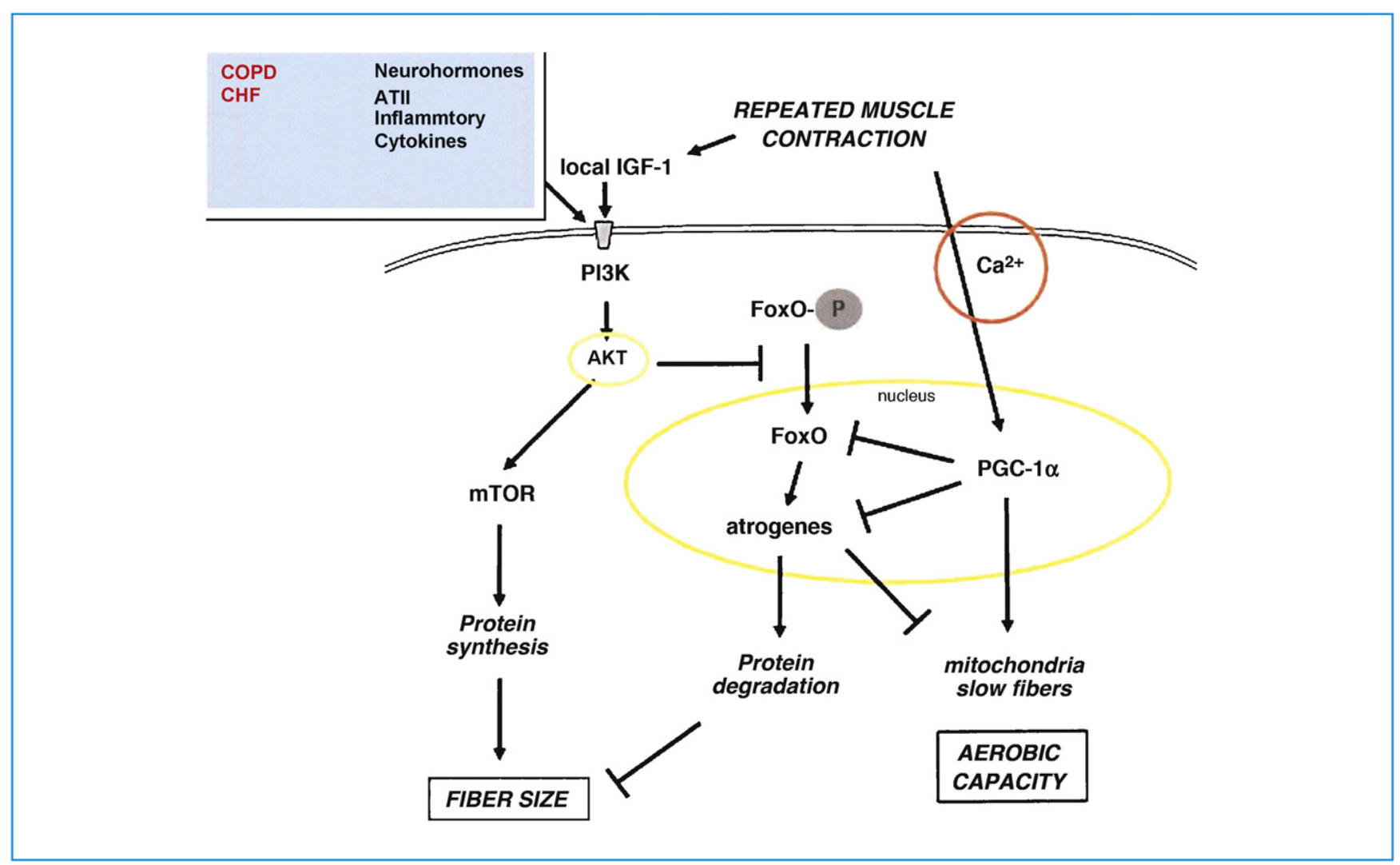

Figure 4 Common Signaling pathways in the regulation of fiber type and size in different pathological situations.

\section{Catabolic-anabolic imbalance}

There are several lines of evidence that metabolism in COPD and $\mathrm{CHF}$ is altered. An activation of catabolic pathways, together with a decreased anabolic capacity, has been reported. Plasma levels of catabolic hormones (cortisol, catecholamines, and angiotensin II) are increased in these conditions, whereas levels of anabolic hormone (dehydroandrosterone) are decreased. This is accompanied by the development of resistance towards growth hormone and insulin. Indices of catabolic-anabolic imbalance, such as the cortisol/dehydroandrosterone ratio, correlate with the prognosis of the disease and the degree of muscle wastage [38].

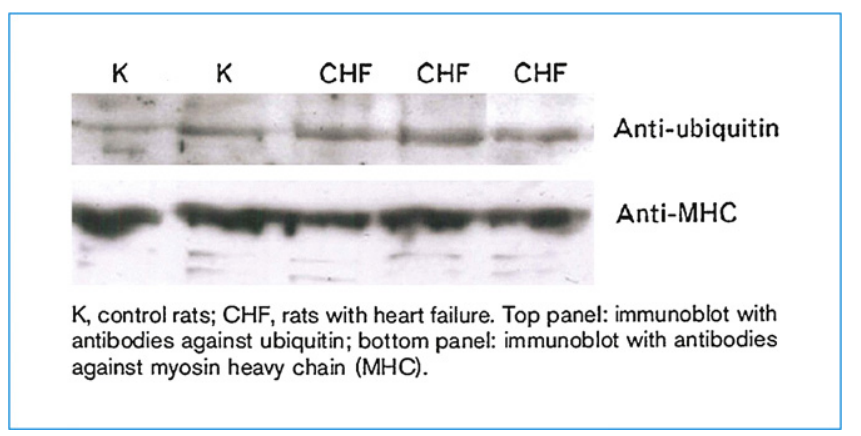

Figure 5 Myosin Ubiquitinization in Skeletal Muscle of rats with Heart Failure and muscle wastage.

\section{Neurohumoral activation in COPD}

Dyspnea, respiratory motor drive, and autonomic control are anatomically and functionally coupled tightly in the brainstem. Specifically, the perception of respiratory discomfort is represented in the sensorimotor integration area of the limbic system that governs autonomic control [39], and the central respiratory motor drive is linked with central sympathetic outflow in the brainstem [40]. These central interactions indicate that dyspnea and increased respiratory drive in COPD may be pathophysiologically linked to heightened sympathetic activation, although the complexity of these interactions within the CNS makes it difficult to clearly distinguish cause and effect.

Furthermore, COPD is associated with several homeostatic disturbances that may directly trigger sympathetic activation. Of those mechanisms likely to contribute to neurohumoral activation in COPD, hypoxemia and ergoreflexes are among the most important. Use of $\beta$-agonist medications, obesity, and tobacco smoking might also contribute in individual patients.

\section{Hypoxemia and hypercapnia}

Chronic hypoxia has long been known to trigger a hyperadrenergic state (for review, see Hansen and Sander [41]. Acute exposure to hypoxia also increases microneurographic measures of sympathetic activity [42]. Studies in healthy subjects showed that following hypoxia, sympathetic activation is significant and long lasting and compensates to oppose the hypoxic vasodilator mechanism. In COPD [38], 


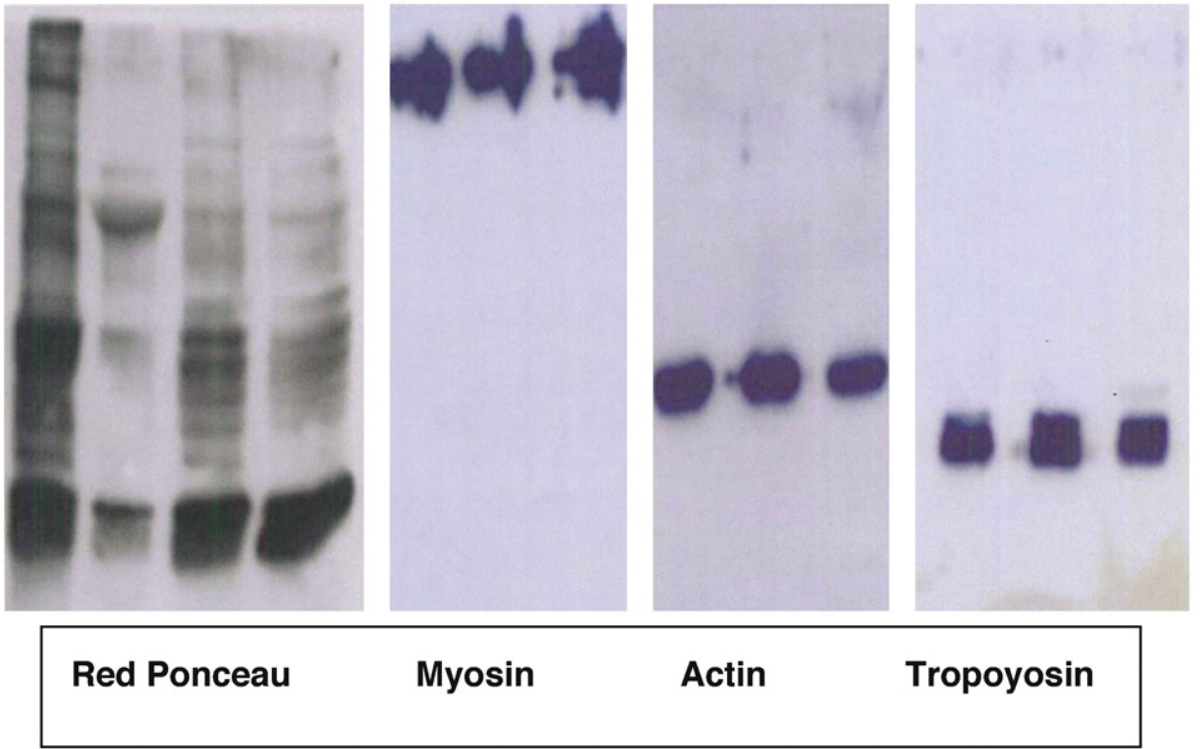

Figure 6 Oxyblot analysis showing peroxidation of myofibrillar proteins in skeletal muscle of patients during development of muscle wastage. Blue dots represent oxydated proteins.

there is evidence of sympathetic activation even in normoxic patients [9], and daytime blood gases do not correlate with sympathetic activation $[43,44]$. However, it is possible that nocturnal hypoxemia might contribute to daytime sympathetic activation, as is thought to be the case in patients with obstructive sleep apnea [44]. Although acute hypercapnia also elicits sympathetic activation, there are no data pointing to a role of chronic hypercapnia in any heightened sympathetic drive in COPD [42].

As a striated muscle, the diaphragm is crucial for breathing. Any negative effect on the contractile properties of the diaphragm, which is already at a mechanical disadvantage in COPD, is detrimental for ventilation and potentially for gas exchange. The effects of sympathetic activation on the diaphragm or accessory respiratory muscles have not been specifically addressed in previous studies. However, it is reasonable to speculate that the negative effects of sympathetic activation on skeletal muscle function also apply to the diaphragm and accessory respiratory muscles.

\section{Protein wasting}

There are at least two pathways by which muscle proteins can be degraded.

\section{Ubiquitin-dependent protein wasting}

Ubiquitin-dependent wastage involves protein ubiquitinization (Fig. 5) and requires activation of the ubiquitin-proteasome system. The process is very similar to that described for cancer cachexia [22]. We have recently demonstrated that fiber atrophy is accompanied by myosin ubiquitinization in a monocrotaline model of right heart failure. Myosin, once bound to ubiquitin (Fig. 4), is taken into the proteasome and degraded. It is therefore clear that this mechanism contributes to the loss of myofibrils and further depresses muscle contractile efficiency. The previously mentioned
IGF1/Akt/FOXO-induced system, which leads to the activation of atrogin-1 (ubiquitin ligase) [24,25], and NF-kB, induced ubiquitin ligase, are two other ubiquitin-dependent protein-wasting pathways.

This latter pathway is mediated by NF-kB activation via a TNF $\alpha-$. NF-kB induces

MuRF1 transcription and the generation of E3 ubiquitin ligases. Locally acting IGF1 inhibits ubiquitin-mediated muscle atrophy in chronic left-ventricular dysfunction, and this is due to blockage of FOXO expression and ubiquitin ligase (atrogin-1; muscle atrophy F-box protein, MAFbx) [22,23].

\section{Ubiquitin-independent protein wasting}

There are three systems involved in ubiquitin-dependent protein wastage: the lysosomal, calpain and caspase systems. The first two are not TNF $\alpha$-related, whereas the last one is TNF $\alpha$-related [41].

\section{Sarcomeric protein oxidation (Fig. 6)}

In addition to the changes described above, there are some other factors that contribute to muscle dysfunction, such as contractile protein oxidation. We have previously shown that muscles with higher levels of oxidation have a depressed peak force generation and slower contraction and relaxation times [19]. I-NOS production and oxidative stress are sufficient to activate NF-kB, a transcription factor for proinflammatory cytokine gene expression that contributes to peripheral muscle damage [37]. In CHF, the presence of oxidized sarcomeric proteins (myosin heavy chain, actin and tropomyosin) positive for oxyblot staining produces a contraction impairment in the whole muscle. Protein oxidation in the skeletal muscle is related to the high oxidative stress, iNOS production and free radical 
formation. Contractile protein oxidation can interfere with contraction and make contraction inefficient $[19,36]$.

\section{Implications and future trends}

This new knowledge of the pathophysiological mechanisms will certainly produce new treatments leading to the improvement of muscle mass, strength and performance. We know that chronic treatment of heart failure and COPD with approved drugs can produce favorable effects on skeletal muscle.

Angiotensin-converting enzyme (ACE) inhibitors, which are known to reduce morbidity, mortality, hospital admissions, and decline in physical function and exercise capacity in congestive heart failure (CHF) patients, have been postulated to have positive effects on skeletal muscle trophic state and function. These may be mediated by direct mechanical, metabolic, anti-inflammatory, nutritional, neurological and angiogenetic action. This has been shown in hypertensive subjects with or without CHF [45].

We have shown that treatment with enalapril and losartan in patients with CHF can restore MHC patterns and improve muscle performance [21]. Beta-blockers, which are very effective in improving the prognosis and quality of life in CHF patients, can prevent skeletal muscle contractile protein peroxidation $[19,46]$. Carnitine has been shown to produce improvements in skeletal muscle contractile protein composition and reduce inflammation, apoptosis and atrophy in experimental models of heart failure [47]. Similarly, stimulation of the GH-IGF-1 axis can cause similar effects in animals [48]. Nutritional intervention may have the potential to obtain substantial improvements in muscle bulk.

Our task for the future will be to determine whether pharmacological and nutritional interventions, together with exercise training, especially in the early stages of the chronic disease, are able to prevent the development of muscle wasting in randomized controlled trials and observational studies.

\section{Conclusions and limitations of this review}

Although this was not the aim of the present review, it is important to fit all of the pathophysiological mechanisms reported above within the general concept of sarcopenia and cachexia occurring in chronic wasting diseases. All of these concepts have been recently reviewed by Muscaritoli et al. [49] in a consensus paper. In this joint document, the relationship between malnutrition, anorexia, and cachexia leading to sarcopenia and their link with chronic diseases, such as COPD and CHF, have been described.

In this paper, we concentrated on muscle wasting, which is the common denominator between cachexia and sarcopenia processes. We focused not only on quantitative changes leading to muscle mass bulk loss but also on the qualitative molecular changes responsible for muscle dysfunction. Qualitative changes are, in our opinion, not completely described by the term sarcopenia, which in its etymology describes loss of muscle mass rather than loss of function. Qualitative and quantitative changes are intimately connected, and it may be possible to envisage interventions at the molecular level targeted to stimulate cell growth, ameliorate altered metabolism and prevent or block wasting in the near future

\section{Conflict of interest}

The authors have no conflict of interest to declare.

\section{References}

[1] Andreas A, Anker S, Scanton P, Somers V. Neurohormonal activation as a link to systemic manifestation of chronic lung disease. Chest 2005;128:3618-24.

[2] Wagner PD. Possible Mechanisms underlying the development of cachexia in COPD. Eur Resp J 2008;31:492-501.

[3] Barreiro E, Schols AMWJ, Polkey MJ. Cytokine profile in quadriceps muscle of patients with severe COPD. Thorax 2008;63:100-7.

[4] Couillard A, Prefaut C. From muscle disuse to myopathy in COPD: potential contribution of oxidative stress. Eur Resp J 2005;26:703-19.

[5] Yende S, Wanderer GW, Tolley EA. Inflammatory markers are associated with ventilatory limitation and muscle dysfunction in obstructive lunge disease in well functioning elderly subjects. Thorax 2006;61:10-6.

[6] Casadevall C, Coronell C, Ramirez Sarmiento AL. Upregulation of pro-inflammatory cytokines in the intercostal muscles of COPD patients. Eur Resp J 2007;30:701-7.

[7] Scalvini S, Volterrani M, Zanelli E, Pagani M, Mazzuero G, Coats AJ, et al. Decreased heart rate variability in patients with chronic obstructive pulmonary disease. Chest 1994;106:1432-7.

[8] Hofford JW, Milakofsky L, Vogel WH, Sacher RS, Savage GJ, Pell S. The nutritional status in advanced emphysema associated with chronic bronchitis: a study of amino acid and catecholamine levels. Am Rev Respir Dis 1990;141:902-8.

[9] Sakamaki F, Satoh T, Nagaya N, Kyotani S, Nakanishi N, Ishida Y. Abnormality of left ventricular sympathetic nervous function assessed by (123). Imetaiodobenzylguanidine imaging in patients with COPD. Chest 1999;116:1575-81.

[10] Dalla Libera L, Zennaro R, Sandri M, Ambrosio GB, Vescovo G. Apoptosis and atrophy in rat slow skeletal muscles in chronic heart failure. Am J Physiol Cell Physiol 1999;277:C982-6.

[11] Vescovo G, Serafini F, Dalta Libera L, Leprotti C, Facchin L, Tenderini $\mathrm{P}$, et al. Skeletal muscle myosin heavy chains in heart failure: correlation between magnitude of the isozyme shift, exercise capacity and gas exchange measurements. Am Heart J 1998;135:130-7.

[12] Vescovo G, Volterrani M, Zennaro R, Sandri M, Ceconi C, Lorusso R, et al. Apoptosis in the skeletal muscle of patients with heart failure: investigation of clinical and biochemical changes. Heart 2000;84:431-7.

[13] Dalla Libera L, Vescovo G. Muscle wastage in chronic heart failure, between apoptosis, catabolism and altered anabolism: a chimaeric view of inflammation? Curr Opin Clin Nutr Metab Care 2004; 7:435-41.

[14] Lindsay DC, Lovegrove CA, Dunn MJ, Bennett JG, Pepper JR, Yacoub $\mathrm{MH}$, et al. Histological abnormalities of diaphragmatic muscle may contribute to dyspnea in heart failure. Circulation 1992;86(suppl I):I515 (Abstract).

[15] Coats AJS, Clark AL, Piepoli M, Volterrani M, Poole-Wilson PA. Symptoms and quality of life in heart failure. The muscle hypothesis. Br Heart J 1994;72:36-9.

[16] Vescovo G, Ravara B, Gobbo V, Angeilni A, Dalla Libera L. Skeletal muscle fibres synthesis in heart failure: role of PGC1a, calcineurin and GH. Int J Cardiol 2005;104:298-306. 
[17] Musarò A, McCullagh KJ, Naya FJ, Olson EN, Rosenthal N. IGF-1 induces skeletal myocyte hypertrophy through calcineurin in association with GATA-2 and NF-Atc1. Nature 1999;400:581-5.

[18] Dalla Libera L, Ravara B, Volterrani M, Gobbo V, Della Barbera M, Angelini A, et al. Beneficial effects of GH/IGF-1 on skeletal muscle atrophy and function in experimental heart failure. Am J Physiol Cell Physiol 2004;286:C139-44.

[19] Dalla Libera L, Ravara B, Gobbo V, Danieli Betto D, Germinario E, Arigelini A, et al. Skeletal muscle myofibrillar protein oxidation in heart failure and the protective effect of Carvedilol. J Mol Cell Cardiol 2005;38:803-7.

[20] Vescovo G, Zennaro R, Sandri M, Carraro U, Leprotti C, Ceconi C, et al. Apoptosis of skeletal muscle myofibers and interstitial cells in experimental heart failure. J Mol Cell Cardiol 1998;30: 2449-59.

[21] Vescovo G, Dalla Libera L, Serafini F, Leprotti C, Facchin L, Volterrani $M$, et al. Improved exercise tolerance after losartan and enalapril in heart failure: correlation with changes in skeletal muscle myosin heavy chain composition. Circulation 1998;98:1742-9.

[22] Chamberlain JS. Cachexia in cancer zeroing in on myosin. N Engl J Med 2004;351:2124-5.

[23] Puigserver P, Spiegelman BM. Peroxisome proliferators-activated receptor-g coactivator $1 \mathrm{a}$ (PGC-1 $\alpha$ ): transcriptional coactivator and metabolic regulator. Endocr Rev 2002;24:78-90.

[24] Sandri M, Sandri C, Gilbert A, Skurk C, Calabria E, Picard A, et al. Foxo transcription factors induce the atrophy-related ubiquitin ligase atrogin-1 and cause skeletal muscle atrophy. Cell 2004;117:399-412.

[25] Pallafacchina G, Calabria E, Serrano AL, Kalhovde JM, Schiaffino S. A protein kinase B dependent and rapamycin sensitive pathway controls skeletal muscle growth but not fiber type specification. Proc Natl Acad Sci USA 2002;99:9213-8.

[26] Schulze PC, Fang J, Kassik KA, Gannon J, Cupesi M, MacGillivray C, et al. Transgenic overexpression of locally acting insulin-like growth factor-1 inhibit ubiquitin-mediated muscle atrophy in chronic left-ventricular dysfunction. Circ Res 2005;97:418-26.

[27] McKinnell IW, Rudnicki MA. Molecular mechanisms of muscle atrophy. Cell 2004;119:907-10.

[28] Skurk C, Izumiya Y, Maatz H, Razeghi P, Shiojima I, Sandri M, et al. The $\mathrm{FOXO} 3$ a transcription factor regulates cardiac myocyte size downstream of AKT signaling. J Biol Chem 2005;280: 20814-23.

[29] Musarò A, McCullagh K, Paul A, Houghton L, Dobrowolny G, Molinaro $M$, et al. Localized Igf-1 transgene expression sustains hypertrophy and regeneration in senescent skeletal muscle. Nat Genet 2001;27:195-200.

[30] Musarò A, Giacinti C, Borsellino G, Dobrowolny G, Pelosi L, Cairns $L$, et al. Stem cell mediated muscle regeneration is enhanced by local isoform of insulin-like growth factor 1 . Proc Natl Acad Sci USA 2004;101:1206-10.

[31] Lin J, Wu H, Tarr PT, Zhang CY, Wu Z, Boss O, et al. Transcriptional co-activator PGC-1a drives the formation of slow twitch muscle fibres. Nature 2002;418:797-801.

[32] Volterrani M, Clark AL, Ludman PF, Swan JW, Adamopoulos S, Piepoli $M$, et al. Predictors of exercise capacity in chronic heart failure. Eur Heart J 1994;15:801-9.
[33] Krown KA, Page MT, Nguyen C, Zechner D, Gutierrez V, Comstock KL, et al. Tumor necrosis factor (-induced apoptosis in cardiac myocytes. Involvement of the sphingolipids signalling cascade in cardiac cell death. J Clin Invest 1996;98:2854-65.

[34] Llovera M, Garcia Martinez C, Agell N, Lopez-Soriano FJ, Argiles JM. TNF can directly induce the expression of ubiquitin-dependent proteolytic system in rat soleus muscles. Biochim Biophys Res Commun 1997;230:238-41.

[35] Attaix D, Ventadour S, Codran A, Béchet D, Taillandler D, Combaret L. The ubiquitin-proteasome system and skeletal muscle wasting. In: Mayer M, Layfield R, editors. Essays in biochemistry, 41. London: Portland Press; 2005. p. 173-86.

[36] Tsutsui H, Ide T, Hayashidani S, et al. Enhanced generation of reactive oxygen species in the limb skeletal muscles from a murine infarct model of heart failure. Circulation 2001;104: 134-6.

[37] Mann D, Reid M. Exercise training and skeletal muscle inflammation in chronic heart failure: feeling better about fatigue. J Am Coll Cardiol 2003;42:869-72.

[38] Anker S, Sharma R. The syndrome of cardiac cachexia. Int J Cardiol 2002;85:51-66.

[39] Shields Jr RW. Functional anatomy of the autonomic nervous System. J Clin Neurophysiol 1993;10:2-13.

[40] Zhong S, Zhou SY, Gebber GL, et al. Coupled oscillators account for the slow rhythms in sympathetic nerve discharge and phrenic nerve activity. Am J Physiol 1997;272:R1314-2.

[41] Hansen J, Sander M. Sympathetic neural overactivity in healthy humans after prolonged exposure to hypobaric hypoxia. J Physiol 2003;546:921-9.

[42] Somers VK, Mark AL, Zavala DC, et al. Influence of ventilation and hypocapnia on sympathetic nerve responses to hypoxia in normal humans. J Appl Physiol 1989;67:2095-100.

[43] Calbet JA. Chronic hypoxia increases blood pressure and noradrenaline spillover in healthy humans. J Physiol 2003;551: $379-86$.

[44] Somers VK, Anderson EA, Mark AL. Sympathetic neural mechanisms in human hypertension. Curr Opin Nephrol Hypertens 1993;2:96-105.

[45] Onder G, Vedova CD, Pahor M. Effects of ACE inhibitors on skeletal muscle. Curr Pharm Des 2006;12(16):2057-64.

[46] Vescovo G, Ravara B, Dalla Libera L. Skeletal muscle myofibrillar protein oxidation and exercise capacity in heart failure. Basic Res Cardiol 2008;103(3):285-90.

[47] Vescovo G, Ravara B, Gobbo V, Sandri M, Angelini A, Della Barbera M, et al. L-Carnitine: a potential treatment for blocking apoptosis and preventing skeletal muscle myopathy in heart failure. Am J Physiol Cell Physiol 2002;283(3):C802-10.

[48] Dalla Libera L, Ravara B, Volterrani M, Gobbo V, Della Barbera M, Angelini $A$, et al. Beneficial effects of GH/IGF-1 on skeletal muscle atrophy and function in experimental heart failure. Am J Physiol Cell Physiol 2004;286(1):C138-44.

[49] Muscaritoli M, Anker SD, Argilés J, Aversa Z, Bauer JM, Biolo G, et al. Consensus definition of sarcopenia, cachexia and pre-cachexia: joint document elaborated by Special Interest Groups (SIG) "cachexia-anorexia in chronic wasting diseases" and "nutrition in geriatrics". Clin Nutr 2010 Apr;29(2): 154-9. 